\title{
CREDIBILIDAD DE LOS MEDIOS EN ESPAÑA: DIVERGENCIAS DE PERCEPCIÓN Y CARACTERIZACIÓN DE LOS ESCÉPTICOS
}

\author{
News media credibility in Spain: differences in \\ perception and characterization of the skeptics
}

\section{Sergio Roses y Bernardo Gómez-Calderón}

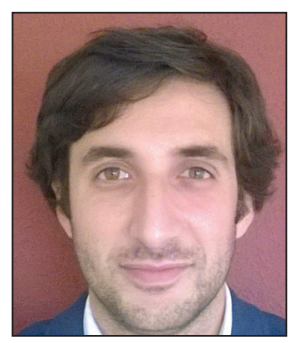

Sergio Roses es profesor del Departamento de Periodismo de la Universidad de Málaga. Sus investigaciones abordan la cultura profesional, formación y situación laboral de los periodistas con perspectiva comparada. También estudia la confianza de los ciudadanos en los medios. Ha publicado en revistas como Journalism studies, Comunicar, Communication \& society, Journalism \& mass communication educator o Estudios sobre el mensaje periodístico, entre otras.

http://orcid.org/0000-0003-3264-6888

sergioroses@uma.es

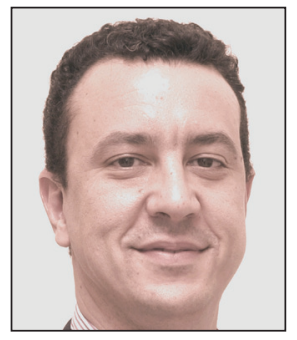

Bernardo Gómez-Calderón es profesor contratado doctor del Departamento de Periodismo de la Universidad de Málaga. Investiga en las áreas de géneros periodísticos, periodismo especializado y estructura de la comunicación. Cuenta con más de 60 publicaciones científicas entre artículos, libros y capítulos de libro. Fue coordinador del Informe anual de la profesión periodística (20052011).

http://orcid.org/0000-0002-9245-9251

bjgomez@uma.es

Universidad de Málaga, Facultad de Ciencias de la Comunicación Campus de Teatinos, s/n. 29071 Málaga, España

\section{Resumen}

Se examinan las actitudes de los españoles hacia los medios de comunicación para conocer en qué medida la población es crítica con ellos. Se pretende establecer posibles predictores que caractericen a los individuos más escépticos ante la labor de los medios. A partir de una encuesta de alcance nacional, se concluye que los españoles confían en los medios de forma global, si bien no consideran que sean imparciales, y se obtiene una serie de rasgos que diferencian a los ciudadanos más escépticos, que tienden a ser personas de género masculino y edad madura, con un nivel de formación alto, habitantes de grandes ciudades en la mitad norte del país, que más bien desconfían de las relaciones interpersonales y que consumen menos televisión que la media.

\section{Palabras clave}

Medios de comunicación; Credibilidad; Noticias; Escepticismo ante los medios; Audiencias; Encuestas; España.

\begin{abstract}
The aims of this article are twofold: to examine attitudes towards news media in Spain in order to measure to what extent consumers are critical about them, and to establish possible predictors that characterize the individuals who are the most skeptical. A national survey showed that, in general, Spaniards trust their media, although they do not consider them to be impartial; it also revealed a set of typical characteristics of the more incredulous media consumer group: they are mainly middle-aged men with a high level of education living in large cities in the north of Spain who are distrustful towards interpersonal relationships and watch less TV than the average.
\end{abstract}

\section{Keywords}

Mass media; Credibility; News media; Media skepticism; Audiences; Surveys; Spain. 
Roses, Sergio; Gómez-Calderón, Bernardo (2015). “Credibilidad de los medios en España: divergencias de percepción y caracterización de los escépticos". El profesional de la información, v. 24, n. 4, pp. 432-439.

http://dx.doi.org/10.3145/epi.2015.jul.10

\section{Introducción}

En cualquier sociedad hay sectores de la audiencia que confían de modo general en los medios y otros, menos numerosos, que se muestran escépticos ante sus mensajes. Estos últimos constituyen grupos de usuarios que, como establece Tsfati (2003), resultan menos influenciables que el resto y acusan en menor medida los efectos de la comunicación masiva.

En el caso de España no abundan los estudios empíricos centrados en la credibilidad que el público atribuye a los medios o en las distintas actitudes que éstos generan entre la audiencia. Por ello y con ánimo exploratorio, este trabajo se propone los siguientes objetivos:

a) determinar en qué medida la población española es crítica con los medios;

b) caracterizar a aquellos individuos que menos credibilidad depositan en los contenidos periodísticos.

\subsection{El concepto de credibilidad}

La credibilidad ha representado tradicionalmente la meta más alta a la que aspiran los medios de comunicación, que asumen que gozar de la confianza de la audiencia redunda en beneficios tangibles -ingresos- e intangibles - prestigio social, identificación-(Meyer, 2004; Oyedeji, 2009). De acuerdo con la mayor parte de las investigaciones, la credibilidad se concibe como el resultado de un proceso en el que el individuo examina y evalúa subjetivamente las cualidades de un emisor, contenido, fuente o cualquier otra instancia informativa. Es decir, se entiende como una variable actitudinal, resultado de un proceso cognitivo (Gunther, 1992; Austin; Dong, 1994; Slater; Rouner, 1996; Choi; Watt; Lynch, 2006; Meyen; Schwer, 2007).

La credibilidad de los medios es, para los autores de referencia, el resultado aditivo de un conjunto de factores o cualidades. Gaziano y McGrath (1986) establecen 12 items definitorios:

- fair;

- unbiased;

- tells the whole story;

- accurate;

- respects people's privacy;

- watch after readers'/viewers' interests;

- concerned about the community's well-being;

- separate fact and opinion;

- can be trusted;

- concerned about the public interest;

- is factual;

- has well-trained reporters.

Por su parte, Meyer (1988) diferencia dos dimensiones en el concepto de credibilidad: believability y community affiliation, medidas a través de nueve pares de adjetivos tomados en su mayoría de Gaziano y McGrath (en el segundo grupo sólo se añade is patriotic como rasgo definitorio). En un nivel de aplicabilidad más amplio, Johnson y Kaye (2000, p. 868) consideran que la credibilidad de los medios depende de cuatro propiedades: imparcialidad, veracidad, precisión y profundidad.

Por su carácter sincrético (es decir, conciliador de puntos de vista distintos), frente a otras propuestas y por su frecuente utilización en el ámbito académico (Hellmueller; Trilling, 2012), consideramos esta última visión la más útil en el marco de nuestra investigación.

La credibilidad es el resultado de un proceso en el que el individuo examina y evalúa subjetivamente las cualidades de un emisor, contenido, fuente $u$ otra instancia informativa

\subsection{Variables que inciden en la actitud hacia los me- dios}

A escala internacional hay numerosos trabajos que analizan posibles predictores de la credibilidad entre la audiencia, aunque ninguno se ha ocupado hasta ahora del caso español.

Centrándonos en las variables contempladas en este estudio, la mayoría de los autores apunta a que los hombres, los individuos de mayor edad, los de mayor poder adquisitivo y los mejor formados tienden a ser más críticos con los medios (Mulder, 1981; ASNE, 1985; Robinson; Kohut, 1988; Eveland; Shaw, 2003; Tsfati; Ariely, 2013). También se ha hallado que las personas con menos confianza interpersonal recelan en mayor medida de las noticias (Jackob, 2012; Tsfati; Ariely, 2013).

Respecto a la ideología política, estudios circunscritos a Estados Unidos han detectado mayor escepticismo hacia los medios entre los simpatizantes de los partidos conservadores (Bennet; Rhine; Flinckinger, 2001; Eveland; Shah, 2003; Tsfati; Capella, 2003; Jones, 2004; Lee, 2005; 2010; The Pew Research Center, 2005; Gronke; Cook, 2007), aunque en el análisis de otros países, como Suecia, los resultados obtenidos son diametralmente opuestos (Tsfati; Ariely, 2013, p. 11).

También se ha evidenciado una correlación positiva entre credibilidad y exposición a los medios, de modo que quien utiliza un canal concreto suele atribuirle más credibilidad que los que recurren a él sólo de forma esporádica (Peng, 2005; Zhang, 2006; Armstrong; Collins, 2009; Choi; Watt; Lynch, 2006; Mehrabi; Hassan; Ali, 2009). Asimismo, parece existir mayor tendencia a confiar en los contenidos perio- 
dísticos entre las personas que tienen un alto consumo de televisión (Jackob, 2010; Tsfati; Ariely, 2013).

\subsection{El caso de España}

El sistema social en el que se relacionan medios e individuos determina las actitudes de estos últimos frente a los mensajes periodísticos. Por ello, más allá de las particularidades de los receptores, en el análisis de la credibilidad hay que tener en cuenta también cómo están estructurados los emisores en cada contexto comunicativo concreto.

El sistema de medios español se ajusta al modelo "pluralista polarizado" (Hallin; Mancini, 2008), caracterizado por:

- posicionamientos ideológicos claros (Bustamante, 2006; León-Gross; Gómez-Calderón, 2011; Humanes et al., 2013);

- un papel activo del Estado en el sector audiovisual (Bustamante, 2006; Rodero-Antón; Sánchez-Serrano, 2007; Medina; Ojer, 2009);

- un desarrollo asimétrico de los emisores comerciales;

- un insuficiente grado de profesionalización del periodismo (González-Cortés; Paniagua-Rojano, 2008).

La dieta mediática de los españoles ha sido definida tradicionalmente como pobre en contenidos informativos y muy enfocada al audiovisual. España es el cuarto país de la Unión Europea en consumo de televisión, pero el octavo por la cola en cuanto a lectura de prensa escrita (Comisión Europea, 2013). Existen marcadas diferencias geográficas: en las comunidades septentrionales, los porcentajes de lectura de diarios y de escucha de radio informativa son más altos, y el tiempo dedicado al visionado de televisión es menor (Farias-Batlle, 2011). Asimismo, la población española se caracteriza por una baja competencia mediática (Ferres et al., 2011, p. 167).

En cuanto a las actitudes, según el Centro de Investigaciones Sociológicas (CIS, 2013), la audiencia desconfía de los medios como institución aunque no tanto de las informaciones que suministran, siendo más críticos con los contenidos políticos y económicos, tendencias constatadas también en otros estudios (Farias-Batlle, 2006-2011). Los encuestados señalan que la cualidad que les lleva a decantarse por unos medios u otros es su "imparcialidad y objetividad" (43,5\%), y aunque en conjunto consideran que se encuentran altamente "politizados" (Farias-Batlle, 2011), se muestran satisfechos ante la fiabilidad y profundidad con la que informan (CIS, 2013).

Pero más allá de estas constataciones generales, y de algunos estudios como el de Calvo-Porral et al. (2014), limitado a la prensa, en España carecemos de información sobre eventuales diferencias entre unos grupos de individuos y otros en relación con la credibilidad que les inspiran los medios, así como sobre las características que definen a los receptores más críticos con su labor.

\section{Metodología}

Los datos para nuestra investigación se recabaron a través de una encuesta telefónica asistida por ordenador (CATI). La población de referencia la constituyeron las personas mayores de edad residentes en España, 38.159.410 de acuerdo con el INE . Se usó una muestra probabilística estratificada por afijación proporcional de acuerdo con las características geográficas, de edad y sexo del universo. La selección de los individuos se realizó aleatoriamente a partir del listín telefónico. La muestra final se compuso de 1.200 entrevistas, con un intervalo de confianza del $2,83 \%$ y un nivel de confianza del $95 \%(p=q=50)$. La encuesta se realizó durante la primera semana del mes de junio de 2014. Las entrevistas tuvieron una duración media de 11 minutos.

Para determinar la variable criterio, se adoptaron las cuatro propiedades de Johnson y Kaye (2000) antes citadas: imparcialidad, veracidad, precisión y profundidad, con respuestas tipo Likert de 5 puntos (acuerdo-desacuerdo).

Se elaboró un índice aditivo a partir de los cuatro items, a los que para evitar sesgos se había aplicado una rotación aleatoria. Se probó la unidimensionalidad a partir de un análisis factorial exploratorio de componentes principales y rotación Varimax. El índice KMO resultó meritorio $(0,85)$ y la prueba de esfericidad de Bartlett, significativa $(p<0,05)$. El determinante de la matriz de correlaciones $(>0,00001)$ descartó las excesivamente elevadas. Los cuatro items pertenecían a una única dimensión, "credibilidad", que explicaba el $82,54 \%$ de la varianza. Posteriormente, se examinó la fiabilidad. El estadístico Cronbach de Alfa alcanzó un valor demasiado elevado (Alfa $>0,90$ ), luego alguno de los items era redundante. De acuerdo con los estadísticos total-elemento, la eliminación de "profundidad" reducía Alfa hasta un valor excelente de 0,89 . Se extrajo dicho ítem para favorecer la idoneidad del instrumento. Finalmente, se comprobó la validez convergente con la variable "confianza en los medios" al hallar correlación directa entre las dos variables $(r=0,39 . p<0,01)$.

Las variables independientes contempladas fueron las siguientes:

- tamaño de hábitat

$1=$ hasta 2.000 habitantes

$2=$ mayor de 2.000 hasta 10.000

$3=$ mayor de 10.000 hasta 50.000

$4=$ mayor de 50.000 hasta 100.000

$5=$ mayor de 100.000 hasta 250.000

$6=$ más de 250.000

- $\operatorname{sexo}($ mujer= 1);

- edad (años);

- nivel formativo (escala de 1 a 6, siendo 1 "sin estudios" y 6 "titulado superior/doctor/máster");

- autoposición política (escala de 0 a 10, donde 0 es extrema izquierda y 10 extrema derecha);

- confianza interpersonal (necesitas tener mucho cuidado con la gente= 1);

- medio preferido para informarse

$1=$ prensa en papel $u$ online

$2=$ gratuitos

$3=$ radio

$4=$ televisión

$5=$ redes sociales

$6=$ revistas

- frecuencia de consumo de noticias de televisión (escala de 1 a 5 , siendo 1 "nunca" y 5 "prácticamente todos los días"); 
- frecuencia de consumo de noticias de prensa en papel u online (escala de 1 a 5 , siendo 1 "nunca" y 5 "prácticamente todos los días");

- interés por la información política (escala de 1 a 5 , siendo 1 "nada" y 5 "mucho").

A continuación se especifican las características de la muestra obtenida:

- edad media de los individuos: 48,20 años $(\mathrm{Dt}=16,99)$;

- 50,5\% mujeres;

- 26,5\% titulados universitarios;

- 70,5\% con algún grado de desconfianza interpersonal;

- 41,4\% interesados poco o nada en información política;

- mayoría de individuos más bien de izquierdas $(M=4,43$. $\mathrm{Dt}=2,37)$;

- 77,5\% seguidores de noticias en televisión "prácticamente todos los días";

- 26,6\% lectores diarios de periódicos en papel u online.

La cuestión que despierta más desconfianza es la de la imparcialidad, único ítem donde los escépticos son mayoría

\section{Resultados}

\subsection{Percepción de la credibilidad de los medios}

El análisis de las frecuencias indica que las puntuaciones extremas de la escala fueron señaladas en menor proporción que las centrales. No obstante, podemos reseñar la existencia de cierto grado de escepticismo hacia la labor de los emisores informativos (tabla 1). La actitud negativa se extiende entre aproximadamente un 19 y un 34\% de la población, según el aspecto a tratar. Si no se toman en consideración las posiciones neutrales, la cuestión que despierta más desconfianza es la de la imparcialidad, único ítem donde los escépticos son mayoría. En el resto, el porcentaje de personas que aprueba el quehacer de los medios supera al de los críticos.

En promedio, la población percibe la credibilidad de los medios como "regular" aunque la tendencia apunte mínimamente hacia una actitud positiva ( $M=3,09$ en una escala de 1 a 5 puntos. Dt=0,82). De nuevo se observa una excesiva centralidad en la valoración de los encuestados. Puede que una gran parte haya optado por expresar "ni acuerdo ni desacuerdo" ante lo abstracto del objeto de referencia, ya que los medios constituyen un ecosistema heterogéneo. En cualquier caso, más de dos tercios de la muestra sí lo hizo aunque no señalara mayoritariamente las puntuaciones extremas de las escalas. A pesar de ello, quienes se decantan por percibir los medios como "creíbles" son más numerosos que quienes desaprueban su labor informativa.

Adicionalmente, se acometió un análisis de conglomerados bietápico para hallar los clústeres en que podía dividirse a la población respecto a sus actitudes, utilizando los tres items del constructo de credibilidad. Se usó Log-verosimilitud como medida de distancia y el criterio de conglomeración bayesiano de Schwarz (BIC). Los resultados identificaron la existencia de tres grupos (gráfico 1), siendo buena la calidad del conglomerado (medida de silueta de cohesión y separación $>0,5)$.

El grupo más numeroso (55,1\% de la muestra) estaba constituido por aquellos que mostraban desacuerdo con que los medios son imparciales $(M=2,75$. $D t=0,64)$ y acuerdo moderado con que informan con veracidad $(M=3,13$. $D t=0,43)$ y precisión $(\mathrm{M}=3,26$. $\mathrm{Dt}=0,51)$.

En promedio, la población percibe la credibilidad de los medios como "regular" aunque la tendencia apunte mínimamente hacia una actitud positiva

El segundo grupo más amplio (24,3\%) estaba formado por quienes manifestaron bastante grado de acuerdo con que los medios informan con imparcialidad $(M=4,23$. $D t=0,43)$, veracidad $(M=4,21$. $D t=0,41)$ y precisión $(M=4,15$. $\mathrm{Dt}=$ $0,49)$.

Finalmente, un 20,5\% componían el grupo más crítico, con un alto grado de desacuerdo con la imparcialidad $(M=1,88$.

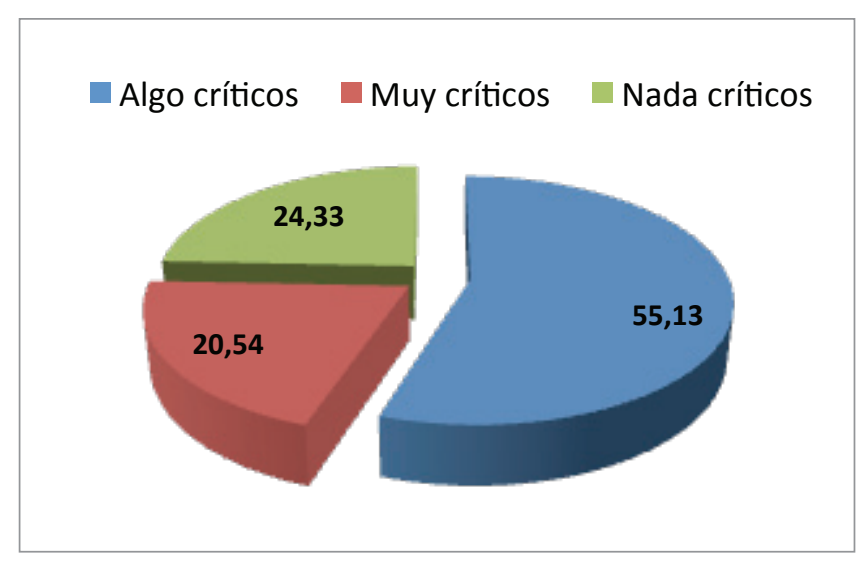

Grafico 1. Grupos de individuos según su percepción sobre la labor de los medios (\%) 
$\mathrm{Dt}=0,38)$, la veracidad $(\mathrm{M}=1,93 . \mathrm{Dt}=0,36)$ y la precisión $(\mathrm{M}=$ 2,03 . $\mathrm{Dt}=0,48$ ) de los emisores informativos. Este colectivo es el que más adelante tratamos de caracterizar.

Mediante la prueba Anova de un factor constatamos además que las medias de los grupos diferían de forma clara. Las puntuaciones promedio de los items resultaron significativamente diferentes para:

- imparcialidad [F de Welch $(2,659,80)=2206,89$. $p=0,000]$;

- precisión [F de Welch $(2,557,08)=1251,38 . p=0,000]$;

- veracidad [F de Welch $(2,576,59)=2332,32 . p=0,000]$.

\subsection{Relación entre variables: caracterizando a los más críticos}

Para determinar los rasgos individuales presentados por los españoles con una percepción negativa sobre la credibilidad de los medios, se acometió un análisis de regresión múltiple sobre el índice aditivo de credibilidad y otro discriminante sobre los dos conglomerados que presentaban las percepciones más extremas (nada críticos/muy críticos) con el fin de diferenciar a los encuestados de la forma más precisa posible.

Tabla 2. Predictores de la credibilidad ${ }^{3}$

\begin{tabular}{|l|l|c|c|c|}
\hline Modelo & & $\begin{array}{c}\text { Coeficientes } \\
\text { tipificados }\end{array}$ & t & Sig. \\
\hline & & Beta & & \\
\hline 1 & (Constante) & & 13,148 & 0 \\
\hline & Es mujer=1 & 0,058 & 2,0170 & $0,044^{*}$ \\
\hline & Edad & $-0,112$ & $-3,521$ & $0,000^{*}$ \\
\hline & Nivel formativo & $-0,159$ & $-4,870$ & $0,000^{*}$ \\
\hline & Desconfía de la gente=1 & $-0,094$ & $-3,326$ & $0,001^{*}$ \\
\hline & Más bien de derechas=1 & 0,063 & 2,202 & $0,028^{*}$ \\
\hline & Extremistas políticos=1 & $-0,040$ & $-1,431$ & 0,153 \\
\hline & Frecuencia con que ve noticias en TV & 0,137 & 4,691 & $0,000^{*}$ \\
\hline & Frecuencia con que escucha noticias en radio & 0,026 & 0,782 & 0,434 \\
\hline & Prefiere la tele=1 & 0,149 & 3,279 & $0,001^{*}$ \\
\hline & Prefiere la radio=1 & 0,080 & 1,991 & $0,047^{*}$ \\
\hline & Prefiere la prensa=1 & 0,064 & 1,604 & 0,109 \\
\hline & Tamaño de hábitat & $-0,097$ & $-3,369$ & $0,001^{*}$ \\
\hline & Vive en el Sur ${ }^{*}=1$ & 0,086 & 3,017 & $0,003^{*}$ \\
\hline
\end{tabular}

Tabla 3. Variables discriminantes entre los grupos "Nada críticos" y “Muy críticos" ante la labor de los medios

\begin{tabular}{|l|c|c|c|}
\hline $\begin{array}{c}\text { Variables ordenadas por importancia } \\
\text { a la hora de definir los grupos }\end{array}$ & $\begin{array}{c}\text { Coeficientes canóni- } \\
\text { cos tipificados }\end{array}$ & $\begin{array}{c}\text { Nada críticos } \\
\text { (media) }\end{array}$ & $\begin{array}{c}\text { Muy críticos } \\
\text { (media) }\end{array}$ \\
\hline Nivel formativo & 0,47 & 3,44 & 4,00 \\
\hline Frecuencia noticias TV & $-0,44$ & 4,76 & 4,47 \\
\hline Desconfía de la gente=1 & 0,40 & 0,61 & 0,75 \\
\hline Prefiere la TV para informarse=1 & $-0,39$ & 0,60 & 0,44 \\
\hline Vive en el sur=1 & $-0,35$ & 0,54 & 0,35 \\
\hline Tamaño hábitat & 0,34 & 3,77 & 4,37 \\
\hline Edad & 0,26 & 48,19 & 48,22 \\
\hline $\begin{array}{l}\text { Lambda de Wilks= 0,84. P=0,000. Chi cuadrado= 89,60. P=0,000 } \\
\text { Casos correctamente clasificados= 67,2\% }\end{array}$ & & \\
\hline
\end{tabular}

En la regresión lineal múltiple ${ }^{2}$ se incluyeron trece variables en una misma ecuación a fin de dilucidar cuáles eran realmente predictores de la credibilidad de los medios (tabla 2). El modelo de regresión hipotetizado resultó estadísticamente significativo $[F(13,1153)=9,697 . p=0,000]$. Sin embargo, el R cuadrado alcanzó tan sólo el valor de 0,099 y los predictores seleccionados explicaron sólo un $10 \%$ de la varianza.

Como se observa en la tabla 2 , sólo hay tres variables no significativas. Por el contrario, el modelo advierte la relevancia de las otras diez. El nivel formativo y los dos predictores relacionados con el consumo televisivo fueron los más importantes.

Se realizó a continuación el análisis discriminante de conglomerados mediante el método Lambda de Wilks. Nuestro interés residía en la caracterización de los casos extremos, de modo que seleccionamos exclusivamente los grupos "muy crítico" y "nada crítico". Se convirtió la variable en dummy (1= muy crítico con los medios) y se añadió a la función como variable de agrupación.

Los resultados (autovalor de 0,189, correlación canónica de 0,399 ) indicaron que existe una única función discriminante que permite de forma significativa $(p=0,000)$ clasificar modestamente a los sujetos en los grupos de "nada críticos" y "muy críticos". El análisis desveló que probablemente no todas las variables eran discriminantes $y$, por consiguiente, se debía determinar la influencia de cada una en la función obtenida. Tras testarlas mediante la opción "usar método de inclusión por pasos", se obtuvo que de las 13 variables contempladas originalmente en la ecuación, sólo 7 resultaban útiles para discriminar a los grupos (tabla 3), quedando excluidas las relacionadas con el sexo, la ideología política y el consumo de prensa escrita y de radio. Las variables depuradas presentaron valores adecuados de tolerancia de entre 0,798 y 1 . El modelo clasificó correctamente al $67,2 \%$ de los casos originales agrupados, lo cual implica un grado de confianza en las predicciones bueno.

De los resultados se deduce que el nivel formativo y la frecuencia con que se siguen las noticias de televisión eran las variables más importantes a la hora de diferenciar a los grupos, siendo en este caso la edad la variable menos relevante. En cualquier caso, las personas 
"muy críticas" con los medios tenían mayor nivel formativo que las "nada críticas", seguían en menor medida las noticias de televisión y preferían otros canales para informarse, vivían en mayor proporción en poblaciones más grandes y en el norte de la península y eran de mayor edad, características todas ellas coincidentes con las obtenidas en el análisis de regresión múltiple.

\section{Conclusiones}

Los resultados apuntan a que actualmente la población española mantiene una postura poco crítica ante los medios; en este sentido, la tendencia coincide con los hallazgos de Farias-Batlle (2006-2011). No obstante, el análisis individual de los indicadores revela que algunos aspectos del quehacer periodístico despiertan más recelos que otros.

Los españoles perciben claramente en los medios sesgo y paralelismo político, de ahí que valoren peor la imparcialidad que el resto de items contemplados en nuestro estudio. No obstante, llama la atención que el porcentaje de críticos no sea mayor. Quizá se deba a que entre los encuestados destaca un colectivo con alto consumo televisivo: no hay que olvidar que este rasgo se ha revelado en nuestro estudio como uno de los predictores significativos de la credibilidad ${ }^{6}$.

Las personas que confieren menos credibilidad a los medios son de sexo masculino, edad avanzada, nivel formativo alto, mayor grado de desconfianza interpersonal y se definen como de centro o de izquierdas

A pesar de que se piensa que los medios no son imparciales, no se cuestionan la honestidad y la profesionalidad con que se elaboran las noticias, pues se valoran positivamente su veracidad, precisión y profundidad. Por otro lado, apenas una cuarta parte de la muestra contaba con estudios universitarios, y hay que tener en cuenta que, como hemos comprobado, existe una relación inversa entre el nivel formativo y la confianza en los medios, lo cual explicaría en parte el alto grado de conformidad registrado.

Pero la credibilidad percibida por la población ha de entenderse no sólo a partir del funcionamiento de los emisores, sino también de las características personales de los receptores. Por este motivo, nos preguntamos por las variables que podrían predecir las distintas percepciones de la audiencia. De acuerdo con el análisis, las personas que confieren menos credibilidad a los medios son de sexo masculino, edad avanzada, nivel formativo alto y mayor grado de desconfianza interpersonal, al igual que ocurre en otros países. En posicionamiento político, se definen como de centro o de izquierdas, un rasgo anómalo -aunque no exclusivo- en el contexto internacional.

Nuestro trabajo también revela que vivir en poblaciones grandes predispone a ser más crítico, quizá porque la agenda de los medios dominantes se centra en los municipios importantes, careciendo los habitantes de poblaciones más pequeñas de información de primera mano con la que enjuiciar la construcción periodística de la realidad. Del mismo modo que en el Norte los hábitos mediático-culturales son más ricos que en el Sur, las personas residentes en zonas meridionales muestran una actitud menos escéptica que las del resto del país. A falta de posteriores estudios que incorporen nuevas variables, podemos interpretar esta brecha como el efecto de diferencias culturales históricas entre las distintas regiones españolas (Fernández-Alonso, 2010; Campos; Fernández, 2004) o del distinto grado de competencia mediática de sus habitantes (Ferrés et al., 2011).

Los resultados señalan que un nivel de estudios bajo y un consumo informativo vinculado sobre todo a la televisión son factores que predisponen en mayor medida a no cuestionar los medios. Consideramos de relevancia este dato, ya que pone de manifiesto la relación existente entre la formación y los hábitos de consumo y la actitud crítica de los ciudadanos. El uso de la televisión como vía informativa preponderante podría estar llevando a la audiencia a un nivel de conformidad en el que la veracidad de las noticias raramente se cuestiona.

En un sistema pluralista polarizado, donde la separación entre periodismo y política es cuanto menos borrosa, una actitud crítica hacia la información por parte de los ciudadanos constituye un indicador de salud democrática. La resistencia ante el influjo de los medios que muestran aquellos que hemos definido como escépticos, y cuyas características tratamos de establecer aquí, resulta indudablemente beneficiosa. Y sería deseable el desarrollo por parte de la Administración de políticas educativas que incidieran sobre los hábitos mediáticos de la población, con el fin de avivar un espíritu crítico similar al que los escépticos evidencian.

\section{Agradecimientos}

Este estudio forma parte de las investigaciones emprendidas en el marco del Proyecto de Excelencia SEJ-8073 Credibilidad del sistema de medios en Andalucía. Confianza de la ciudadanía en los medios audiovisuales, financiado por la Consejería de Economía, Innovación y Ciencia de la Junta de Andalucía (Resolución de 13 de marzo de 2012).

\section{Notas}

1. Cifra a 1 de enero de 2014.

2. Previamente se realizó el análisis bivariante con cada par de variables. No se da cuenta de él por motivos de espacio. En cualquier caso, desveló la existencia de asociaciones entre variables, por lo que se aplicó el análisis multivariante.

3. Resultado de un análisis de regresión múltiple. Los valores marcados con asterisco señalan qué predictores son significativos. El valor de Beta indica la importancia del predictor y los signos negativos, una relación inversa entre el predictor y la credibilidad percibida. Por ejemplo, en el caso de Edad, cuarto predictor en importancia, el signo negativo señala que cuanto menor es la edad del individuo mayor es la credibilidad que atribuye a los medios.

4. Obtenida a partir de la recodificación de la variable Comunidad Autónoma.

5. Resultado de un análisis discriminante donde el valor de 
los coeficientes canónicos tipificados indica la importancia de cada variable discriminante a la hora de definir los grupos. El valor de Lambda y su significación evidencian que la función calculada contiene variables que sirven para diferenciar a los grupos.

6. La relación directa entre consumo de televisión y confianza en los medios probablemente responda al hecho de que en este canal el paralelismo político resulta menos manifiesto que en otros, al menos en el caso español (Humanes et al., 2013), generando por ello menos recelo entre la audiencia.

\section{Bibliografía}

Armstrong, Cory; Collins, Steve (2009). "Reaching out: newspaper credibility among young adult readers". Mass communication and society, v. 12, n. 1, pp. 97-114. http://dx.doi.org/10.1080/15205430701866592

ASNE (1985). Newspaper credibility: Building readers trust. Washington, DC: MORI Research, ISBN: 9789995288396

Austin, Erica; Dong, Qingwen (1994). "Source v. content effects on judgments of news believability". Journalism quarterly, v. 71, n. 4, pp. 973-983.

http://dx.doi.org/10.1177/107769909407100420

Bennet, Stephen; Rhine, Staci; Flinckinger, Richard (2001). “Assessing Americans' opinion about the news media's fairness in 1996 and 1998". Political communication, v. 18, n. 2, pp. 163-182.

http://dx.doi.org/10.1080/105846001750322961

Bustamante, Enrique (2006). "Hacia un servicio público democrático". En: Díaz-Nosty, Bernardo (dir.). Medios de comunicación. Tendencias'06. Madrid: Fundación Telefónica, pp. 357-362. ISBN: 8489884684

http://www.infoamerica.org/TENDENCIAS/tendencias/ tendencias06/pdfs/26.pdf

Calvo-Porral, Cristina; Martínez-Fernández, Valentín-Alejandro; Juanatey-Boga, Óscar (2014). "Credibilidad de los medios de comunicación: análisis de la prensa diaria desde el comportamiento del consumidor". El profesional de la información, v. 23, n. 3, pp. 300-309.

http://dx.doi.org/10.3145/epi.2014.may.10

Campos, Miryam; Fernández, Itziar (2004). “Diferencias culturales entre País Vasco, Galicia, Centro y Sur de España. Investigaciones antropológicas y un estudio empírico retrospectivo". Revista vasca de sociología y ciencia política, n. 38, pp. 103-134.

http://goo.gl/nT3qxm

CIS (2013). Estudio 2981. Barómetro marzo 2013.

http://www.analisis.cis.es/cisdb.jsp

Choi, Junho; Watt, James; Lynch, Michael (2006). "Perceptions of news credibility about the war in Iraq: Why war opponents perceived the internet as the most credible medium". Journal of computer-mediated communication, n. 12, pp. 209-229.

http://dx.doi.org/10.1111/j.1083-6101.2006.00322.x

Comisión Europea (2013). Eurobarómetro 80.

http://goo.gl/jRLrzU
Eveland, William; Shah, Dhavan (2003). "The impact of individual and interpersonal factors on perceived news media bias". Political psychology, v. 24, n. 1, pp. 101-117. https://www.journalism.wisc.edu/ dshah/PP2003.pdf http://dx.doi.org/10.1111/0162-895X.00318

Farias-Batlle, Pedro (dir.) (2006-2011). Informe anual de la profesión periodística. Madrid: Asociación de la Prensa de Madrid.

http://www.apmadrid.es/publicaciones/informe-anual-dela-profesion-periodistica

Fernández-Alonso, Mercedes (2010). "Valores y creencias en el proceso de modernización”. Papers, v. 95, n. 4, pp. 1031-1051.

http://goo.gl/onYIBT

Ferrés-Prats, Joan; García-Matilla, Agustín; Aguaded-Gómez, José-Ignacio; Fernández-Cavia, Josep; Figueras, Mònica; Blanes, Magda (2011). Competencia mediática. Investigación sobre el grado de competencia de la ciudadanía en España. Madrid: Ministerio de Educación, ITE.

http://ntic.educacion.es/w3//competencia_mediatica/3_ evaluacion/7.htm

Gaziano, Cecilie; McGrath, Kristin (1986). "Measuring the concept of credibility". Journalism quarterly, n. 63, pp. 451-462. http://www.aejmc.org/home/wp-content/uploads/2012/09/ Journalism-Quarterly-1986-Gaziano-451-621.pdf

González-Cortés, María-Eugenia; Paniagua-Rojano, Francisco-Javier (2008). "Las asociaciones de la prensa españolas, un modelo de organización profesional consolidado". Revista latina de comunicación social, n. 63, pp. 435-444. http://dx.doi.org/10.4185/RLCS-63-2008-793-435-444

Gronke, Paul; Cook, Timothy E. (2007). “Disdaining the media: The American public's changing attitudes toward the news". Political communication, v. 24, n. 3, pp. 259-281. http://dx.doi.org/10.1080/10584600701471591

Gunther, Albert (1992). "Biased press or biased public? Attitudes toward media coverage of social groups". The public opinion quarterly, v. 56, n. 2, pp. 147-167.

Hallin, David; Mancini, Paolo (2008). Sistemas mediáticos comparados. Tres modelos de relación entre los medios de comunicación y la política. Barcelona: Hacer. ISBN: 978 8496913127

Hellmueller, Lea; Trilling, Damian (2012). "The credibility of credibility measures: A meta-analysis of credibility research in communication journals, 1951 to 2011". En: The World Association of Public Opinion Research (Wapor) Conf. Hong Kong. http://wapor2012.hkpop.hk/doc/papers/ConcurrentSessionsV/ VD/ND-3.pdf

Humanes, María-Luisa; Montero-Sánchez, María-Dolores; Molina-De-Dios, Ramón; López-Berini, Alfredo (2013). "Pluralismo y paralelismo político en la información televisiva en España". Revista latina de comunicación social, n. 68, pp. 566-581.

http://dx.doi.org/10.4185/RLCS-2013-990

Jackob, Nikolaus (2010). "No alternatives? The relationship between perceived media dependency, use of alternative 
information sources and general trust in mass media". International journal of communication, n. 4, pp. 589-606. http://ijoc.org/index.php/ijoc/article/view/615/435

Jackob, Nikolaus (2012). "The tendency to trust as individual predisposition- exploring associations between interpersonal trust, trust in the media and trust in institutions". Communications, v. 37, n. 1, pp. 99-120.

http://dx.doi.org/10.1515/commun-2012-0005

Johnson, Thomas J.; Kaye, Barbara K. (2000). "Using is believing: The influence of reliance on the credibility of online political information among politically interested internet users". Journalism \& mass communication quarterly, v. 77 n. 4, pp. 865-879.

http://dx.doi.org/10.1177/107769900007700409

Jones, David A. (2004). "Why Americans don't trust the media: A preliminary analysis". International journal of press/ politics, n. 9, pp. 60-75.

http://dx.doi.org/10.1177/1081180X04263461

Lee, Tien-tsung (2005). "The liberal media myth revisited: an examination of factors influencing perceptions of media bias". Journal of broadcasting \& electronic media, v. 49, n. 1, pp. 43-64.

http://dx.doi.org/10.1207/s15506878jobem4901_4

Lee, Tien-tsung (2010). "Why they don't trust the media: An examination of factors predicting trust". American behavioral scientist, v. 54, n. 1, pp. 8-21.

http://dx.doi.org/10.1177/0002764210376308

León-Gross, Teodoro; Gómez-Calderón, Bernardo (2011). "La tertulia en España: Medios públicos, última frontera de la pluralidad". Estudios sobre el mensaje periodístico, n. 17, pp. 67-80.

http://revistas.ucm.es/index.php/ESMP/article/view/36946

Medina-Laverón, Mercedes; Ojer-Goñi, Teresa (2009). “Valoración del servicio público de televisión. Comparación entre la BBC y TVE". Revista latina de comunicación social, n. 64, pp. 275-299.

http://dx.doi.org/10.4185/RLCS-64-2009-823-275-299

Mehrabi, Davood; Hassan, Musa-Abu; Ali, MuhamadSham-Shahkat (2009). "News media credibility of the Internet and television". European journal of social sciences, n. 11, n. 1, pp. 136-148.

http://psasir.upm.edu.my/5622/1/European_Journal_of_ Scientific_Research_11.1.151.pdf

Meyen, Michael; Schwer, Katja (2007). “Credibility of media offering in centrally controlled media systems: a qualitative study bases on the example of East Germany". Media, culture \& society, n. 29, pp. 284-303.

http://dx.doi.org/10.1177/0163443707074260

Meyer, Philip (1988). "Defining and measuring credibility of newspapers: Developing an index". Journalism quarterly, v. 65, n. 3, pp. 567-574.

http://dx.doi.org/10.1177/107769908806500301

Meyer, Philip (2004). "The influence model and newspaper business". Newspaper research journal, v. 25, n. 1, pp. 50-62. http://goo.gl/FS1SBr

Mulder, Ronald (1981). "A log-linear analysis of media credibility". Journalism quarterly, v. 58, n. 4, pp. 635-638. http://dx.doi.org/10.1177/107769908105800420

Oyedeji, Tayo (2009). "A multivariate test of the influence model". International journal on media management, v. 11, n. 2, pp. 72-80.

http://dx.doi.org/10.1080/14241270902763746

Peng, Zhenjung (2005). "Ideology and source credibility: partisan perception bias in believability of CNN, Fox News and PBS". En: Association for Education in Journalism and Mass Communication Conference. San Antonio, Texas. http://goo.gl/hUXPJR

Robinson, Michael J.; Kohut, Andrew (1988). "Believability and the press". Public opinion quarterly, v. 52, n. 2, pp. 174-189. http://dx.doi.org/10.1086/269093

Rodero-Antón, Emma; Sánchez-Serrano, Chelo (2007). "Radiografía de la radio en España". Revista latina de comunicación social, n. 62, pp. 275-299.

http://goo.gl/T8zKfJ

Slater, Michael D.; Rouner, Donna (1996). "How message evaluation and source attributes may influence credibility assessment and belief change". Journalism quarterly, v. 73, n. 4, pp. 974-991.

http://comm 705.wikispaces.com/file/view/Slater++Message+Credibility.pdf

http://dx.doi.org/10.1177/107769909607300415

The Pew Research Center (2005). Trends 2005. Washington DC: The Pew Research Center.

http://www.pewresearch.org/files/old-assets/trends/ trends2005.pdf

Tsfati, Yarif (2003). "Does audience skepticism of the media matter in agenda setting?". Journal of broadcasting \& electronic media, v. 47, n. 2, pp. 157-176.

http://www.hevra.haifa.ac.il/ comm/he/files/yariv/jobem. $p d f$

http://dx.doi.org/10.1207/s15506878jobem4702_1

Tsfati, Yarif; Ariely, Gal (2013). “Individual and contextual correlates of trust in media across 44 countries". Communication research, v. 41, n. 6, pp. 760-782.

http://dx.doi.org/10.1177/0093650213485972

Tsfati, Yarif; Cappella, Joseph (2003). "Do people watch what they do not trust? Exploring the association between news media skepticism and exposure". Communication research, v. 30, n. 5, pp. 504-529.

http://dx.doi.org/10.1177/0093650203253371

Zhang, Mingxin (2006). "The present situation and analysis of mass media use $\&$ media credibility in countriside of midChina: the case of Hubei Province". China media research, v. 2, n. 4, pp. 37-47.

http://www.chinamediaresearch.net/index.php/backissues?id=38 

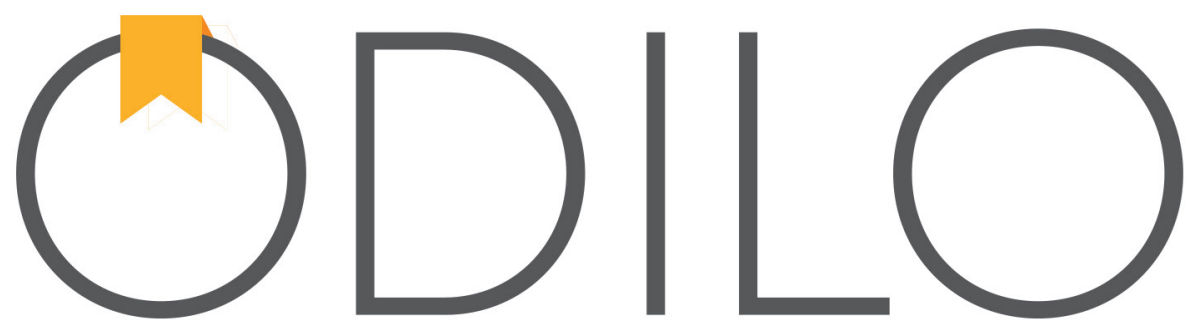

\section{TK}

OdiloTK es una plataforma que permite la gestión bibliotecaria y el préstamo de contenido digital (libro electrónico, audio y video) cumpliendo con los derechos de autor.

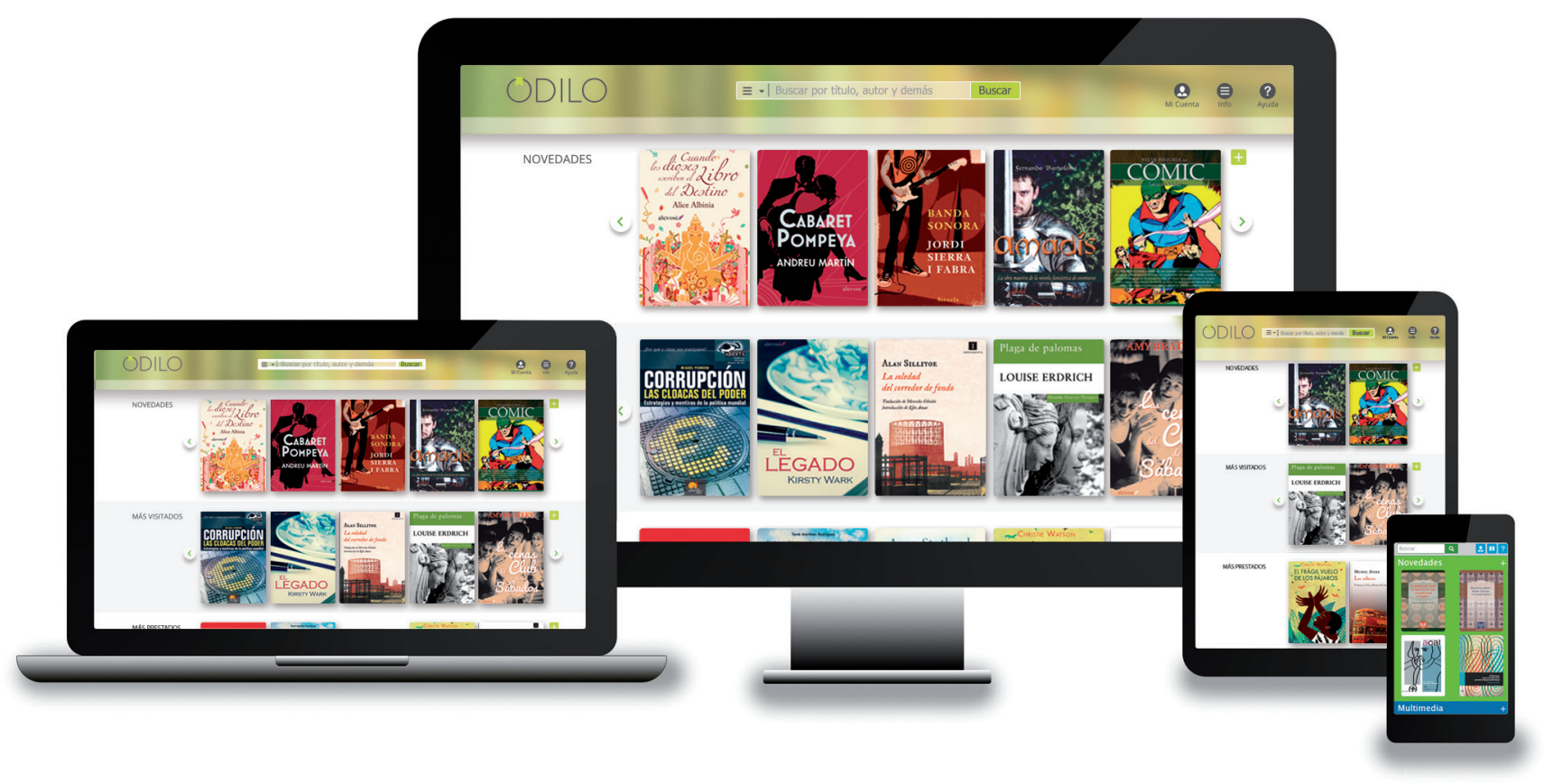

\section{Funcionalidades y puntos claves de la plataforma:}

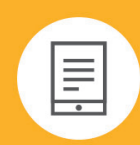

Préstamo de contenido digital en un solo click

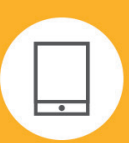

Aplicaciones móviles Odilo: incorpora la tecnología de Adobe, lo que permite que los usuarios accedan al contenido con las credenciales de su biblioteca, sin tener que darse de alta en una cuenta de Adobe.

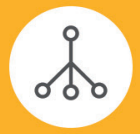

Integración con otros sistemas de gestión bibliotecaria

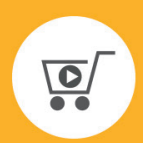

Interconexión con una tienda de contenido digital: permite la entrega inmediata de contenidos

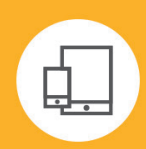

Interfaz atractiva y multidispositivo

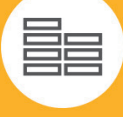

Lectura en streaming:

lectura online a traves de

la aplicación desarrollada por Odilo: Nubereader.

Compatible con el navegador de Kindle.

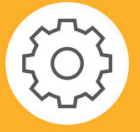

Área de administración avanzada

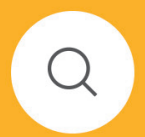

Búsquedas: por facetas por campos principales y avanzada

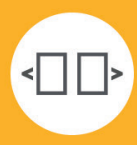

Página de acceso a través de carruseles táctiles y configurables

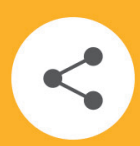

Funcionalidades sociales: sistema de recomendación de contenido, compartición en redes sociales, comentarios, creación de listas de libros para compartir. 